\title{
Nodule Margin Conspicuity
}

National Cancer Institute

\section{Source}

National Cancer Institute. Nodule Margin Conspicuity. NCI Thesaurus. Code C157662.

A description about the radiologic visiblity of a nodule's margins. 